\title{
Impact of Physiotherapy Rehabilitation Program on Post-Operative Stiffness of Supraspinatus Tendinitis
}

\author{
Madhumita Yadav¹, Nupur Thombare², Waqar M. Naqvi³ ${ }^{3}$ Ashish W. Bele
}

${ }^{1}$ Department of Physiotherapy, Ravi Nair Physiotherapy College, Datta Meghe Institute of Medical Sciences, Wardha, Maharashtra, India. ²Department of Physiotherapy, Ravi Nair Physiotherapy College, Datta Meghe Institute of Medical Sciences, Wardha, Maharashtra, India. ${ }^{3}$ Department of Community Health Sciences and Vice

Principal, Ravi Nair Physiotherapy College, Sawangi Meghe, Wardha, Maharashtra, India. ${ }^{4}$ Department Of

Community Health Sciences, Ravi Nair Physiotherapy College, DMIMS (DU), Wardha, Maharashtra, India.

\section{INTRODUCTION}

In the general population, shoulder pain is a common musculoskeletal problem. Older people are frequently affected, and the most common causes of shoulder pain are the problems with rotator cuffs which are seen in primary care practice. The incidence of rotator cuff tear increases with advanced age and it has been estimated that the prevalence of shoulder pain in older patients ranges from $21 \%$ to $27 \%$. $^{(1)}$

Tendons are thick, fibrous tissues that transmits muscle-generated force to the bones and thus generates joint movement. Repeated activities and complex muscletendon overuse can damage tendons that lead to pain and decrease function. (2)

The supraspinatus muscle is a part of the rotator cuff of shoulder.(3) The inflammation of a muscle tendon causes tendinitis and it has varying levels of pain. Supraspinatus tendonitis is an inflammation of supraspinatus tendon often associated with shoulder impingement syndrome.(4) The Supraspinatus tear that causes the supraspinatus tendon to affect the acromion leading to pain is the cause of Supraspinatus Tendinitis. The main cause of the Supraspinatus tear is constant pressure, increased workload and wear and tear associated with age.(5)

Total loss of active abduction at the glenohumeral joint is due to a complete tear of supraspinatus.(6) Supraspinatus tendonitis complications include leading to a rotator cuff tear. Initially, rotator cuff tears were almost identified as secondary to tendon haemorrhage and tendonitis products of untreated impingement.(7)

The primary purpose of rehabilitation to achieve short-term and long-term goal, patient education and consistent plan of care. The patient's awareness on the need to take non-operative steps, improvement, and the availability of choices will assist them in being active participants in their treatment.(7)

\section{KEY WORDS}

Supraspinatus Tendinitis, Supraspinatus Tear, Shoulder Stiffness, Shoulder Pain, Shoulder Rehabilitation
Corresponding Author:

Waqar M. Naqvi,

Ravi Nair Physiotherapy College,

Datta Meghe Institute of Medical Sciences,

Wardha, Maharashtra, India.

E-mail: waqar.naqvi@dmimsu.edu.in

DOI: $10.14260 /$ jemds/2020/522

How to Cite This Article:

Yadav M, Thombare N, Naqvi WM, et al. Impact of physiotherapy rehabilitation program on post-operative stiffness of supraspinatus tendinitis. J Evolution Med Dent Sci 2020;9(33):2403-2405, DOI: 10.14260/jemds/2020/522

Submission 10-05-2020,

Peer Review 06-07-2020,

Acceptance 14-07-2020,

Published 17-08-2020.

Copyright (C) 2020 JEMDS. This is an open access article distributed under Creative Commons Attribution License [Attribution 4.0 International (CC BY 4.0)] 


\section{PRESENTATION OF CASE}

A 60 years-old male presented with a complaint of right shoulder pain since 11 months. The onset was gradual. There was no history of fall or trauma. On anterolateral aspect of right shoulder pain was present. On abduction and flexion of the right shoulder pain was sharp and deep. Pain reported by patient on VAS scale of $6 / 10$. It was made worse by lying on back, reaching and raising with the right arm or taking extended rest. Medicines were used to relieve pain and keeping shoulder adducted. Then he approached to AVBRH where medications (Cartigen, T. Dolocide, Idrofos and Calcium supplements) were given for one month but the pain was not relieved, so he was advised for sonography. The reports showed supraspinatus muscle tear. Then he was advised surgery. He undergone Surgery on $25^{\text {th }}$ January 2019.

After taking informed consent of the patient physical examination was performed. On General examination patient was conscious, co-operative and well oriented with time, place and person and was comfortable in supine and sitting position. He was afebrile, Pulse rate was 64 beats/min and respiratory rate was 24 breaths/min. Abdomino-thoracic type of breathing pattern. Pain assessment was done. The pain was $3 / 10$ on vas when rest and 5/10 during hand movement. The site of pain was on the anterior and posterior shoulder near to glenohumeral joint. The nature of pain was pulling type of pain the onset of the pain was gradual.

General condition of patient was endomorphic; muscle wasting was not present when compared to normal limb, mild swelling on the right shoulder was present and bandage was not present. Attitude of limb was assessed in sitting position, in anterior view shoulder was depressed and retracted. Bony contour prominent over right shoulder when compared with normal. Scars where present over right shoulder.

On palpation, tenderness was present on his right deltoid, infraspinatus, supraspinatus and teres muscles and the grade was 2. Spasm was present on the anterior and posterior part of right shoulder. Tightness on the anterior part of right shoulder was present on examination, upper extremity bilateral deep tendon reflexes were normal and sensation examination also normal. Bilateral lateral flexion, rotation and flexion of cervical spine were normal and pain free. Although, by the neck examination the right shoulder pain could not be reproduced.

\begin{tabular}{|ccccc|}
\hline \multicolumn{2}{|c}{ Right Shoulder } & \multicolumn{2}{c|}{ Left Shoulder } \\
Active & Passive & Action & Active & Passive \\
$0-130$ & $0-135$ & Flexion & $0-180$ & $0-180$ \\
$0-30$ & $0-35$ & Extension & $0-60$ & $0-60$ \\
$0-100$ & $0-110$ & Abduction & $0-180$ & $0-180$ \\
$0-40$ & $0-45$ & Medial Rotation & $0-80$ & $0-80$ \\
$0-50$ & $0-52$ & Lateral Rotation & $0-90$ & $0-90$ \\
\hline \multicolumn{4}{c}{ Table 1 Range of Motion } \\
\hline
\end{tabular}

\begin{tabular}{|ccc|}
\hline Muscle & Measurement (Right) & Measurement (Left) \\
Deltoid & $41.0 \mathrm{~cm}$ & $41.5 \mathrm{~cm}$ \\
Biceps & $40.0 \mathrm{~cm}$ & $40.5 \mathrm{~cm}$ \\
Triceps & $39.0 \mathrm{~cm}$ & $39.5 \mathrm{~cm}$ \\
Brachioradialis & $33.0 \mathrm{~cm}$ & $33.5 \mathrm{~cm}$ \\
\hline \multicolumn{3}{|c|}{} \\
\hline \multicolumn{3}{|c|}{ Table 2 Muscle Girth } \\
\hline
\end{tabular}

Special tests such as Empty can test, Hawkins-Kennedy impingement test was positive and Neer impingement test and drop arm test was negative. The patient lower extremity range of motion and strength were adequate for all his daily living according to his age. His uninvolved upper extremity was functional according to his MMT's, and he had no previous problems from weakness in that upper extremity.

\begin{tabular}{|cccc|}
\hline Muscle & Action & Right & Left \\
Anterior Deltoid \& Coracobrachialis & Flexion & $3 \backslash 5$ & $4 \backslash 5$ \\
Latissimus Dorsi, Teres Major, Posterior Deltoid & Extension & $3 \backslash 5$ & $4 \backslash 5$ \\
Middle Deltoid \&Supraspinatus & Abduction & $3 \backslash 5$ & $4 \backslash 5$ \\
Pectoralis Major & Adduction & $3 \backslash 5$ & $4 \backslash 5$ \\
Subscapularis & Medial rotation & $3 \backslash 5$ & $4 \backslash 5$ \\
Infraspinatus \& Teres Major & Lateral rotation & $3 \backslash 5$ & $4 \backslash 5$ \\
\hline Table 3 Manual Muscle Testing & & \\
\hline
\end{tabular}

\section{CLINICAL DIAGNOSIS}

Post-operative stiffness of supraspinatus tendinitis

\section{DISCUSSION OF MANAGEMENT}

Our short-term goal was to decrease pain on vas scale of 3/10, decrease inflammation, increase Range of motion by 5 degree, improve flexibility, improve strength of rotator cuff muscles. Long- term goal was to regain full strength and endurance of the supraspinatus, infraspinatus, teres minor and subscapularis muscle and to achieve full range of motion and also return the patient to full functioning.

Intervention consisted of three phases.

1. The first phase was start after immobilisation. Initially we started mobilization relaxed passive flexion-extension with sling, gravity eliminated assisted abduction, passive range of motion pain-free and below 90 degrees for abduction, gradual progress to active resisted abduction, gentle mobilizations Grade I/II to increase range of motion. We also gave him hot fermentation and IFT to reduce pain.

2. Second Phase was started after completion of phase 1 in which we gave relaxed passive full range of movement exercises for abductors and flexors. To secure total relaxation and proper stabilization, especially during abduction care were taken. We gradually progressed to active or active-assisted exercises and then resisted exercises in which manual resistance or resistance with dumbbells starting with minimal wight were added. We also gave him exercises such as supine cane full flexion exercise to improve range of motion, isometric exercises to improve strength of the muscles, joint mobilisation, range of motion of shoulder against gravity and pendulum exercises were done. Modality such as IFT was used in order to reduce pain and stimulate muscles.

3. In phase three after 12 weeks we continued the exercises that we did in phase 2 but we also added progressive exercises such as light resistance exercises to improve muscle power. PNF diagonals were also done by us. Selfstretching exercises such as crossover stretch for 12 sec 5 times a day was advised to the patient.

\section{Follow-Up and Outcome}

After 12 weeks, patient was again asked to give follow up and physical examination was performed but mild complaints were seen and reported. 10 weeks after surgery he returned 
to his work. The functional recovery was completed at that time. At the end of rehabilitation period the outcome was the passive abduction of right glenohumeral was full ROM but resisted strength was graded 4/5, MMT's were graded at 4/5 and muscle strength were improved, the patient able to perform all his daily living activities with less pain.

\section{DISCUSSION}

This case report indicates high potential for recovery after reconstruction of supraspinatus in an older adult. This case study stresses the value of an appropriate conservative procedure after surgery is attempted in a patient with supraspinatus tendinitis. It can be difficult to treat a patient with supraspinatus tendonitis and needs active coordination, particularly in more complicated cases. The team will include colleagues in imaging, health services and physical therapy to help this patient to achieve the best result.(7)

The patients will to recover fast from this injury played a big part in the rehabilitation. Further complete co-operation of the patient with the therapist is also proved to be useful during treatment. Patience is very important after continuous treatment for more than 12 weeks the patient was able to work in farm as he was a farmer. Progression of the treatment and proper instructions to be followed by the patient including home program proved to be useful.

The recovery following surgery was of this patient proceeded without any complication our major goal to achieve was to decrease pain because reduction in pain helps in increasing range of motion. Throughout the treatment care was taken in order to regain muscle strength because there should be enough strength to perform farming work. For the management of shoulder dysfunction there are many treatment regimens are available but this regime may be useful. In order to know the appropriate course of treatment the biomechanics of shoulder should be evaluated.(7)

\section{CONCLUSIONS}

Only graded exercises can effectively treat a long-standing supraspinatus tendinitis with traumatic aetiology. IFT and mobilization exercises revealed that a completely regenerated supraspinatus tendon was the outcome of the procedure. At the same time, pain in the shoulder decreased slowly and vigorous movement was regenerated, and also physiotherapy plays an integral role in treating patients of supraspinatus tendonitis.

Financial or Other Competing Interests: None.

\section{REFERENCES}

[1] Lin JC, Weintraub N, Aragaki DR. Nonsurgical treatment for rotator cuff injury in the elderly. J Am Med Dir Assoc 2008;9(9):626-32.

[2] Djurovic N, Hraste M, Stanisic L. A unique case of supraspinatus tendonitis after tennis forehand repetitive motions. Journal of Human Kinetics 2010;23(1):115-9.

[3] Supraspinatus tear [Internet]. Physiopedia https://www.physio-pedia.com/Supraspinatus_tear

[4] Sivakumar GK, Vasudevan AC. Supraspinatus tendinitis and physical therapy management. EC Orthopaedics 2018;9(11):797-800.

[5] Supraspinatus tendinitis - about, causes, physiotherapy \& more.

PORTEA https://www.portea.com/physiotherapy/supraspinatus -tendinitis

[6] Joshi J, Kotwal P. Essentials of orthopaedics \& applied physiotherapy. Elsevier Health Sciences 2016: p. 647.

[7] Tapscott DC, Varacallo M. Supraspinatus tendonitis. In: StatPearls [Internet]. Treasure Island (FL): StatPearls Publishing 2020. 